and patient experience during transition. To implement a service development plan and consider how to evaluate the outcome.

Methods This was a service improvement project performed during Feb-July 2018 in a district general hospital. 53 patients with Type 1 diabetes aged 14-19 years were invited to take part. Patients were surveyed about their experiences and views on transition via two patient questionnaires (pre-intervention and 6 months post-implementation of the transition nurse). Qualitative data was also collected via semi-structured interviews.

Results The response rate for the pre and post-intervention questionnaires was $72 \%$ and $66 \%$ respectively. Overall, $73 \%$ of subjects reported one or more anxieties regarding transition to adult diabetes care. The top two reported anxieties were having to be more independent and the possibility of being admitted to an adult ward. The most common reasons for not attending clinic were that appointments 'clashed' with school or were booked too far in advance. The majority of respondents did not want to meet with the diabetes team at school or have 'virtual' appointments. Overall, the young people felt satisfied with the support provided by the transition nurse. Three key themes were identified from the structured interviews, flexibility of services, the need for more support and worry regarding taking on increased responsibility.

Conclusion A specialist transition nurse may lead to improved patient engagement and patient experience during transition. Patients want more flexibility around appointment times and more support throughout transition. Further research is required to look at specific outcome measures such as glycosylated haemoglobin (HbA1c) and hospital admission rates to determine whether the presence of a transition nurse translates into improved outcome measures in the longer term.

\section{P29 CAN EATING DISORDERS IN CHILDREN AND YOUNG PEOPLE PERMANENTLY AFFECT GROWTH AND PUBERTAL DEVELOPMENT?}

1J Neale*, 'S Pais, ${ }^{2} \mathrm{D}$ Nicholls, ${ }^{1,3} \mathrm{~L}$ Hudson. ${ }^{1}$ Department of Child and Adolescent Mental Health, Great Ormond Street Hospital, London, UK; ${ }^{2}$ The Centre for Psychiatry, Imperial College London, London, UK; ${ }^{3}$ General and Adolescent Paediatric Unit, UCL Great Ormond Street Institute of Child Health, London, UK

\subsection{6/bmjpo-2019-RCPCH-SAHM.34}

Aims We aimed to assess what evidence there is that eating disorders in children and young people affect growth and puberty and to what extent this is permanent. Whilst a number of studies on long term physical sequelae of eating disorders in children and young people exist, reports are conflicting, and this literature has never been systematically reviewed.

Methods We systematically reviewed the literature using Pubmed, Embase, PsychINFO and Web of Science to search for studies which looked at the effects of growth and development in participants $<18$ years with an eating disorder.

Results Of 10,404 abstracts, we retrieved 96 articles and included 28 studies. Both cross-sectional and longitudinal studies were identified. Most individual studies were small (included $<100$ participants). Although we found evidence that eating disorders in children and young people are associated with impaired growth and delayed puberty compared to control groups, meta-analysis suggested no significant difference in stature. Impact on growth was found to be permanent in some studies. However, there was a larger body of evidence to suggest that catch-up growth is possible, with weight gain contributing to an acceleration of growth and final adult heights which were not significantly different from controls. In the majority of studies, pubertal delay was not seen at follow up.

Conclusion Children and young people with eating disorders are at risk of permanent effects on growth and reduced final height, though many will have the potential for catch up thus avoiding final stunting. Children and young people with eating disorders should have their growth and physical development regularly reviewed and monitored, and restoration of growth should be a goal in treatment to allow for catch up and prevent stunting. The potential effects on growth should be discussed with patients and their families.

\section{P30 USING LOW TECHNOLOGY HIGH FIDELTY SIMULATION TO ENHANCE CLINICIAN CONFIDENCE IN ADOLESCENT MENTAL HEALTH CARE}

OA Sanwo*, M Dubus. Department of Paediatrics, East Kent Hospitals University NHS FT, Kent, UK

\subsection{6/bmjpo-2019-RCPCH-SAHM.35}

Background In the 2017 Mental Health of Children and Young People in England Report, 15.4\% of children presented to a physical health specialist such as a paediatrician or GP regarding their mental health. An Australian survey found that over a 7-year period, childhood mental health presentations to Emergency Departments increased three times faster than physical health presentations (Hisock et al, 2018). However, GPs and paediatricians receive little formal training on the assessment and management of common mental health presentations. Despite the clinical and educational advantages of simulation, mental health simulation is less commonly utilised (Attoe et al, 2016). Consequently, little is known about the use of simulation to train paediatric and GP trainees in assessment and management of children and adolescents presenting with mental health concerns.

Aim To increase junior doctor confidence in managing common acute paediatric mental health presentations through the development of a paediatric mental health simulation programme.

Method Low-technology, high-fidelity simulation scenarios on self-harm and eating disorders were developed and incorporated into the weekly in-situ simulation programme for paediatric and GP junior doctors trust-wide over 6 months. Confidence in history-taking, psychosocial assessment and management of the adolescents was assessed by self-report before and after the simulation. Each simulation was subsequently debriefed, including discussions on risk assessment and management, signposting to resources and sharing learning points.

Results Following the simulations there was increased clinicianreported confidence in history taking $(33.4 \%$ vs. $82.6 \%)$, psychosocial assessment $(30.4 \%$ vs. $62.5 \%)$ and management $(33.3 \%$ vs. $58.4 \%)$ of adolescents with self-harm. Thematic analysis of qualitative feedback highlighted the desire for further training as well as the benefits of simulation. 
Conclusion As the mental health needs of children and adolescents continue to rise, it is clear there remains an urgent and unmet need for mental health training for clinicians. This data suggests that low-technology high-fidelity simulation is a feasible method of improving clinician confidence in the assessment and management of adolescents with mental health presentations. It is hoped that by developing such programmes, clinicians will be encouraged to cultivate the necessary skills to appropriately assess and manage patients presenting with mental health concerns.

\section{P31 RACISM AND ITS HARMFUL EFFECTS ON NON- DOMINANT RACIAL, ETHNIC YOUTH AND YOUTH- SERVING PROVIDERS: A CALL TO ACTION FOR ORGANIZATIONAL CHANGE}

\begin{abstract}
${ }^{1,2} \mathrm{MV}$ Svetaz ${ }^{*}{ }^{3,4}{ }^{4} \mathrm{~T}$ Coyne-Beasley. ${ }^{1}$ Division of Family and Community Medicine, Hennepin Healthcare System, Minnneapolis, USA; ${ }^{2}$ Leadership Education in Adolescent Health (LEAH) Program, Division of General Pediatrics and Adolescent Health, Minneapolis, USA; ${ }^{3}$ Division Director, UAB Adolescent Medicine, Children's of Alabama, Birmingham, USA; ${ }^{4}$ Past President, Society for Adolescent Health and Medicine, Society for Adolescent Health and Medicine, Chicago, USA
\end{abstract}

\subsection{6/bmjpo-2019-RCPCH-SAHM.36}

Aims Racism can exert negative effects on the self-concepts, health, well-being and life trajectories of both non-dominant racial-ethnic (NDRE) youth and youth-serving providers. In the face of growing nationalism, ethnocentrism, xenophobia and overt expressions of racism, the Society for Adolescent Health and Medicine (SAHM) recognized the critically important need to address the issue of racism and its impact on both NDRE youth and youth-serving providers. SAHM decided to create a guide for organizations involved in clinical care delivery and health professions training and education to promote safe and affirming environments with the goal to create inclusivity during this difficult time.

Methods In developing this position paper, the authors relied on a review of the literature and expert consensus.

Results The Society for Adolescent Health and Medicine endorses the following positions:

- Organizations, providers, researchers, and policymakers should recognize that racism negatively affects the selfconcept, health and well-being, and life trajectories of both NDRE youth and youth-serving providers.

- Organizations should consider and address racism as a form of structural violence.

- Organizations should reaffirm their commitment to justice and equity and actively develop, implement, and evaluate policies and processes to ensure that racism is not embedded systematically.

- Youth-serving organizations should explicitly convey their views against racism and create safe, welcoming spaces for all.

- Organizations should develop, implement, and evaluate interventions at all levels addressing chronic minority stress and vicarious trauma affecting NDRE providers.

- Organizations should develop, implement, and evaluate interventions at all levels addressing chronic minority stress and vicarious trauma affecting NDRE trainees and students.

- Organizations should develop, implement, and evaluate training for providers to routinely explore and address racism with all youth and effectively intervene when they identify affected youth.
- Providers caring for youth should integrate promising interventions to address racism as a part of routine evaluation and in response to identified aggression.

Conclusions In this position paper, SAHM affirms its commitment to foundational moral and ethical principles of justice, equity, and respect for humanity; acknowledges racism in its myriad forms; defines strategies to best promote resiliency and support the health and well-being of NDRE youth, providers, trainees, and students; and provides recommendations on the ways to best effect systemic change.

\section{P33 ANTIBODIES SUGGESTIVE OF IMMUNITY TO HEPATITIS B AND A ARE NOT DETECTED IN THE MAJORITY OF ADOLESCENTS AND YOUNG ADULTS SEEKING MEDICATION-ASSISTED TREATMENT FOR OPIOID USE DISORDER}

${ }^{1,2}$ E McKnight* ${ }^{3}$ S Stull, ${ }^{1,2}$ S Matson, ${ }^{1,2,4} \mathrm{~A}$ Bonny. ${ }^{1}$ Division of Adolescent Medicine Nationwide Children's Hospital, Columbus, USA; ${ }^{2}$ The Ohio State University, Columbus, USA; ${ }^{3}$ Clinical Pharmacology and Therapeutics Research Branch, NIH/NIDA/IRP, Bethesda, USA; ${ }^{4}$ The Research Instutite at Nationwide Children's Hospital, Columbus, USA

\subsection{6/bmjpo-2019-RCPCH-SAHM.37}

Aims Opioid drugs, particularly morphine and heroin, can result in relative immunosuppression, negatively impacting both innate and acquired immunity. Adolescents and young adults (AYA) with history of injection drug use are at high risk for hepatitis infections; therefore, evaluation of their immune status is imperative. The objective of this study was to document hepatitis immune and infection status among AYA seeking medication-assisted treatment (MAT) for severe opioid use disorder.

Methods A retrospective medical record review was conducted of all adolescent and young adults seeking outpatient MAT for severe opioid use disorder from January 1, 2013 to December 31, 2015. Each chart was assessed for hepatitis A, $\mathrm{B}$, and $\mathrm{C}$ serology testing and prior hepatitis immunization status.

Results Among 193 AYA presenting for MAT who had no evidence of prior hepatitis $\mathrm{B}$ infection, mean age was 19.3 (SD 1.66), 114 (59\%) were female, and 184 (95\%) were white non-Hispanic. Mean age at first opioid use was 15.3 (SD 1.85), and mean age at first heroin use was 17.0 (SD 1.87). Injection drug use and prior incarceration were reported by $136(71 \%)$ and 29 (15\%). Hepatitis C antibody was positive in 66 (34\%). Seropositivity for hepatitis B surface antibody (anti-HBs) and hepatitis A immunoglobulin G (anti-HAV) was $62(32 \%)$ and $45(23 \%)$, respectively. Documentation of hepatitis B immunization initiation was evident in 102 (53\%) and completion in 84 (44\%). Hepatitis A immunization initiation and completion was present in 38 (20\%) and $23(12 \%)$, respectively.

Conclusions Despite recommendationsfor routine vaccination, immunity to hepatitis B and A, as assessed by measurement of anti-HBs and anti-HAV, was not evident in the vast majority of AYA seeking MAT. Documentation of appropriate hepatitis immunization was present in a minority of this high risk population. Further study will explore whether seronegativity to hepatitis $A$ and $B$ is reflective of true lack of immunity, nonimmunization status, or immunosuppression from opioid/heroin misuse in this patient population. 